\title{
Polyphosphonium-based ion bipolar junction transistors
}

\author{
Erik O. Gabrielsson, Klas Tybrandt, and Magnus Berggren ${ }^{\text {a) }}$ \\ Department of Science and Technology, Linköping University, 60174 Norrköping, Sweden
}

(Received 4 July 2014; accepted 17 November 2014; published online 5 December 2014)

\begin{abstract}
Advancements in the field of electronics during the past few decades have inspired the use of transistors in a diversity of research fields, including biology and medicine. However, signals in living organisms are not only carried by electrons but also through fluxes of ions and biomolecules. Thus, in order to implement the transistor functionality to control biological signals, devices that can modulate currents of ions and biomolecules, i.e., ionic transistors and diodes, are needed. One successful approach for modulation of ionic currents is to use oppositely charged ion-selective membranes to form so called ion bipolar junction transistors (IBJTs). Unfortunately, overall IBJT device performance has been hindered due to the typical low mobility of ions, large geometries of the ion bipolar junction materials, and the possibility of electric field enhanced (EFE) water dissociation in the junction. Here, we introduce a novel polyphosphonium-based anion-selective material into npn-type IBJTs. The new material does not show EFE water dissociation and therefore allows for a reduction of junction length down to $2 \mu \mathrm{m}$, which significantly improves the switching performance of the ion transistor to $2 \mathrm{~s}$. The presented improvement in speed as well the simplified design will be useful for future development of advanced iontronic circuits employing IBJTs, for example, addressable drug-delivery devices. @ 2014 AIP Publishing LLC.

[http://dx.doi.org/10.1063/1.4902909]
\end{abstract}

There has been a recent interest in developing diodes ${ }^{1-4}$ and transistors ${ }^{4-8}$ that conduct and modulate ion currents. Such non-linear iontronic components are, for example, interesting as they allow further control of ions in, for instance, electrophoretic drug delivery devices. A range of microfabricated diodes, ${ }^{9-11}$ transistors, ${ }^{12,13}$ and circuits ${ }^{9,14}$ has been constructed using ion-selective membranes. These membranes contain fixed charges of either polarity, compensated by mobile ions of opposite charge (counter-ions). When immersed in an electrolyte, counter-ions can move through the membrane, while ions with the same charge as the fixed charges (co-ions) are repelled. This renders the membrane selective for the counter-ion and can therefore be considered as p- or n-type ion conductors. By combining two membranes of opposite polarity, a bipolar membrane (BM) configuration is obtained ${ }^{15}$ (Figure 1(a)). The BM junction can be biased by an ion current in the reverse and forward directions, respectively. ${ }^{16,17}$ This modulates the ion concentration inside the BM, and thus the ionic conductivity, which then results in an current rectification. ${ }^{2,18}$ In the three-terminal ion bipolar junction transistor ${ }^{12}$ (IBJT), an ion-selective base (B) is connected to oppositely selective emitter (E) and collector (C), forming two BM configurations (EB and BC) (Figure 1(b)). pnp- and npn-IBJTs have been constructed $^{14}$ from photolithography patterned poly(styrene sulfonate) (PSS, p-selective) and quaternized poly(vinylbenzyl chloride) (n-selective) as emitter, collector, and base. In these devices, a neutral poly(ethylene glycol) (PEG) electrolyte is typically inserted into the junction to separate the base from the emitter and collector, ${ }^{12}$ in order to avoid ${ }^{19}$ electric field enhanced (EFE) water dissociation ${ }^{16}$ (Figure 1(a)). EFE water dissociation is typically observed in BMs ${ }^{20}$ and produces water ions inside the BM under reverse bias, which prevents proper IBJT operation. In PEG-IBJTs, the current between the emitter and collector $\left(I_{C}\right)$ is thus modulated by

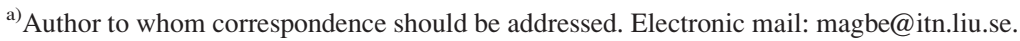


(a)
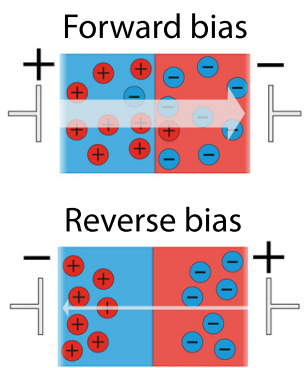

(b)

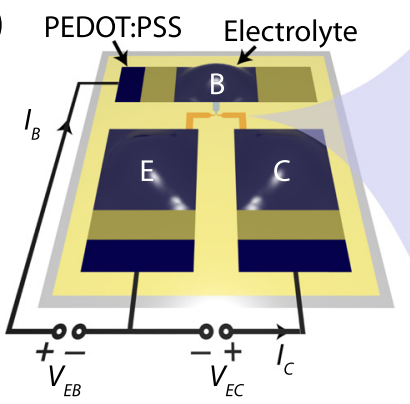

(c)

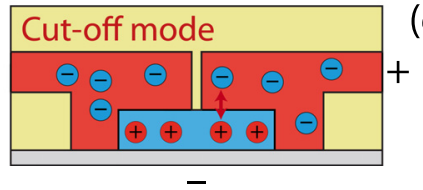

(4) Mobile cation

- Mobile anion

- Anion selective

- Cation selective

Water dissociation
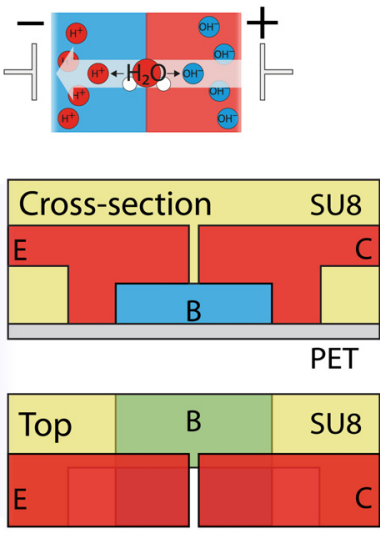

(d)

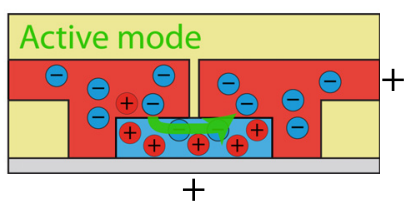

FIG. 1. (a) The modes of operation for a BM; forward bias (high conduction and ion accumulation), reverse bias (low conduction and ion depletion), and EFE water dissociation (high conduction, formation of ions). (b) Illustrations of an npnIBJT, with anion-selective emitter (E) and collector (C) forming a junction with a cation-selective base (B). (c) In cut-off mode, the base and collector extract ions from the junction, prohibiting co-ion migration through the base. (d) In active mode, the forward biased EB injects ions into the base, thus allowing anions from the emitter to migrate as co-ions through the base into the collector.

controlling the ion concentration inside the PEG-junction. ${ }^{21}$ Ions are injected or extracted into the junction depending on the bias of the base $\left(V_{E B}\right)$. In a npn-IBJT, a positive bias is typically applied between emitter and collector $\left(V_{E C}\right)$, thus allowing anions to migrate from the emitter to the collector. In the cut-off mode (Figure 1(c)), a negative bias $V_{E B}$ is applied, resulting in reverse bias of both $\mathrm{EB}$ and $\mathrm{BC}$. Cations in the junction will migrate into the base, while anions will primarily migrate into the collector, due to the higher collector bias. This base current $\left(I_{B}\right)$ will extract ions from the junction, which decreases the ionic conductivity in the junction resulting in a low $I_{C}$. Eventually, the resistive characteristics for ion charge transport, between the emitter and collector, will be entirely dominated by the junction. This gives that most of the applied $V_{E C}$ is consumed across the junction with only a minimal voltage potential drop across the emitter and base terminals.

In the active-mode of the npn-IBJT (Figure 1(d)), the $V_{E B}$ bias at the base is reversed (i.e., now positive). This causes injection of cations, from the base, and anions, from the emitter, into the junction. As the ion concentration increases, anions from the emitter can start to drift across the junction to the collector, thus a high $I_{C}$ is obtained. The high concentration of ions inside the junction is reflected in a low resistive value for ion transport. This now causes the voltage to drop over the emitter and collector terminals, thus lowering the EB forward bias and the injection of ions from the base. At the collector-junction interface, the extraction of anions produces an ion depletion zone and a corresponding voltage drop. Thus, in the active-mode, the applied $V_{E C}$ is primarily consumed across the emitter and collector terminals and also at the collector-junction interface.

The switching speed of an IBJT should be strongly correlated to the distance separating the emitter and collector, ${ }^{14}$ as this length determines the volume that needs to be filled or emptied 
with ions causing modulation of ions in the junction. To achieve a fast-switching IBJT, the junction volume, i.e., the collector-emitter separation, should be as small as possible. However, EFE water dissociation must be avoided since this process ruin the IBJT operation. EFE water dissociation is, in part, driven by the appearance of a large potential drop across a small distance, as occurring at the interface of a BM under reverse bias, producing a high electric field that accelerates the forward reaction rate of water auto-dissociation. ${ }^{16}$ Miniaturization of the collector-emitter distance is therefore problematic, as the separation inside the EB and BC BMs evidently also mush shrink, resulting in higher reverse bias electric fields across the BMs and thus promoting EFE water dissociation. The problem of EFE water dissociation in an IBJT primarily manifests itself in the cut-off mode, as water ions are generated in the reversed biased $\mathrm{EB}$ and BC BMs. These ions produce an elevated cut-off $I_{C}$, and hence deteriorate the IBJTs on-off performance. Here, we report an IBJT, in which the EFE water dissociation is avoided by the use of a novel polyphosphonium-based anion-selective material, which previously has been shown to prevent EFE water dissociation in BM diodes. ${ }^{11}$ This allows the collector and emitter to directly contact the base without an intermediate PEG-layer. Without the need for a PEG-separator inside the BMs, the collector-emitter distance is reduced to only $2 \mu \mathrm{m}$.

Polyphosphonium-based npn-IBJTs were produced following the same manufacturing protocol as was reported for polyphosphonium-based ion diodes. ${ }^{11}$ Conjugated polymer electrodes and cationselective base was patterned from $\sim 200 \mathrm{~nm}$ thick poly(3,4-ethylenedioxythiophene):polystyrene sulfonate film on polyethylene terephthalate-sheets using photolithography and dry-etching. The base was rendered electronically insulating by chemical overoxidation via exposure to sodium hypochlorite through a mask. A $2 \mu \mathrm{m}$ thick SU8-layer was patterned on-top of this configuration, with an opening defining the actual junction. $1 \mu \mathrm{m}$ thick polyphosphonium-based anion-selective emitter and collector were deposited and patterned using photolithography and dry-etching, to overlap with the base at the opening of the SU8. Finally, a second $10 \mu \mathrm{m}$ thick layer of SU8 was used to seal the junction. The membranes were hydrated by incubation in $\mathrm{dH} 2 \mathrm{O}$ for $24 \mathrm{~h}$ before any measurements were carried out. Aqueous $0.1 \mathrm{M} \mathrm{NaCl}$ electrolytes were used during the measurement. All electrical measurements were performed using a Keithley 2602 source meter.

The switching characteristics of the npn-IBJT were obtained by applying $V_{E C}$ of $10 \mathrm{~V}$ and alternating $V_{E B}$ at $\pm 3 \mathrm{~V}$ for various duration of time, see Figure 2 . A periodic $5 \mathrm{~s}$ switching with $8 \mathrm{~Hz}$ measurement rate was used to record the dynamics of the turn-on/off characteristics of the device. When $V_{E B}$ switches from -3 to $+3 \mathrm{~V}$, there is a quick increase in the $I_{B}$, as ions from the base and emitter migrate into the emitter/base junction. After a delay of $\sim 0.25 \mathrm{~s}, I_{C}$ starts to increase due to the increased ion concentration in the emitter/base junction and the subsequent diffusion of anions into the base. As the $I_{C}$ increases, the $I_{B}$ decreases as the voltage drop between the emitter and base decreases, and after $\sim 2 \mathrm{~s} I_{C}$ reaches $90 \%$ of the steady state on-current level. For longer on-switching times, the $I_{B}$ and $I_{C}$ stay stable over $30 \mathrm{~s}$, after which a small increase is observed. This current-drift in both $I_{B}$ and $I_{C}$ is likely due to the contribution of co-ion migration. As cations from the base migrate into the emitter as co-ions, the

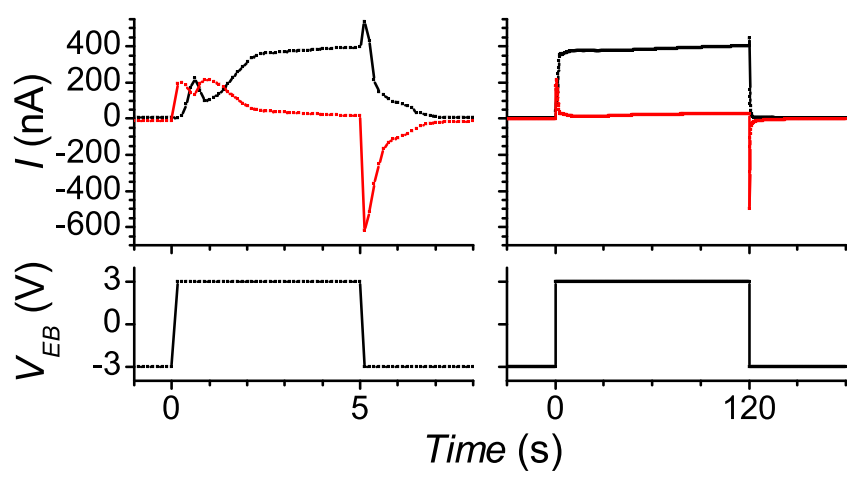

FIG. 2. Emitter-collector current response as the IBJT is switched between cut-off $\left(V_{E B}=-3 \mathrm{~V}\right)$ and active mode $\left(V_{E B}=3 \mathrm{~V}\right)$ for $V_{E C}=10 \mathrm{~V}$, at $5 \mathrm{~s}$ and $120 \mathrm{~s}$ periods. 
conductivity in the emitter increases, leading to an increased $I_{C}$ value. This increases the ion concentration at the base, which gives less selective ion injection and thus more cation injection from the base, i.e., a higher $I_{B}$.

As $V_{E B}$ is switched back to $-3 \mathrm{~V}$, there is a sharp negative peak in $I_{\mathrm{E}}$ as ions are extracted from the junction, which occur mainly through the base (cations) and collector (anions) terminals. As the ion concentration in the base drops, $I_{\mathrm{C}}$ decreases. The transistor turns off to $10 \%$ of the value of the steady state on-current within $\sim 2 \mathrm{~s}$, regardless of the duration of the onstate. The constant turn-off time indicates that ions are not accumulating to a significant extent inside the junction during the on-steady state but are instead constantly transported out of the junction. When all co-ions have been extracted from the junction, the Donnan exclusion prevents subsequent injection of anions into the base, and $I_{\mathrm{C}}$ is therefore low. The on/off ratio of $I_{\mathrm{C}}$ reaches above 100 .

A transfer curve was obtained by scanning $\mathrm{V}_{\mathrm{EB}}$ between -3 and $+3 \mathrm{~V}$ while keeping $V_{E C}$ at $10 \mathrm{~V}$ (Figure 3(a)). As expected, both $I_{\mathrm{C}}$ and $I_{\mathrm{B}}$ remain low for negative $V_{\mathrm{EB}}$. In this range, both $\mathrm{EB}$ and $\mathrm{BC}$ are biased in reverse direction. As $V_{\mathrm{EB}}$ turns positive, the EB configuration is switched into forward bias and ions are injected into the junction. This leads to a linear increase in $I_{\mathrm{C}}$ vs. $V_{\mathrm{EB}}$. For the reverse scan, a minor hysteresis is observed for both the $I_{\mathrm{C}}$ and $I_{\mathrm{B}}$ scans, again probably due to the contribution of co-ion migration due to long time operation of the device.

The transistor output characteristics were obtained by scanning $V_{\mathrm{EC}}$ at different $V_{\mathrm{EB}}$ values (Figure 3(b)). The saturation regime, i.e., the bias mode was both $\mathrm{EB}$ and $\mathrm{BC}$ are in forward bias, was avoided as this has negative impact on the stability of the device. As reported for previous IBJT devices, the output characteristics show a clear saturation behaviour of $I_{\mathrm{C}}$ across the entire range of $V_{\mathrm{EC}}$. Further, the $I_{\mathrm{C}}$ increases linearly with $V_{\mathrm{EB}}$. The increase of both $I_{\mathrm{C}}$ and $I_{\mathrm{B}}$ when operating for extended periods of time in the active mode is again attributed to the addition and inclusion of co-ions in the junction. The current gain $\left(I_{\mathrm{C}} / I_{\mathrm{B}}\right)$ at $V_{\mathrm{EC}}=10 \mathrm{~V}$ decreases with $V_{\mathrm{EB}}$ and reaches $43.9,17.9$, and 10.7 for $V_{\mathrm{EB}}=1 \mathrm{~V}, 2 \mathrm{~V}$, and $3 \mathrm{~V}$, respectively. For higher base bias voltages, the ion concentration increases in the junction and thus the injection selectivity decreases.

In comparison with previously reported IBJTs, ${ }^{12,14,21}$ the lack of a neutral electrolyte layer in the junction has an overall positive effect on the device characteristics. Main performance improvements are found in a decrease in the turn-on time from $9 \mathrm{~s}$ (for npn-IBJT ${ }^{21}$ ) to $2 \mathrm{~s}$, for devices with comparable junction widths and heights. The main contribution to the improved switching speed is likely the decreased length between the emitter and collector. Interestingly, simulations have shown that an extended space charge region (ESCR), for a PEG-IBJT in cutoff mode, can extend several micrometers away from the collector. ${ }^{22}$ Thus, a PEG-IBJT with an emitter-collector separation of single micrometers should show an increased cut-off current due to the ESCR overlapping in the junction. However, by omitting the PEG in the junction, the ESCR is reduced due to screening from the fixed charges in the BM layers. This enables the IBJT, reported here, to operate with retained low cut-off currents. On-off ratios and ion
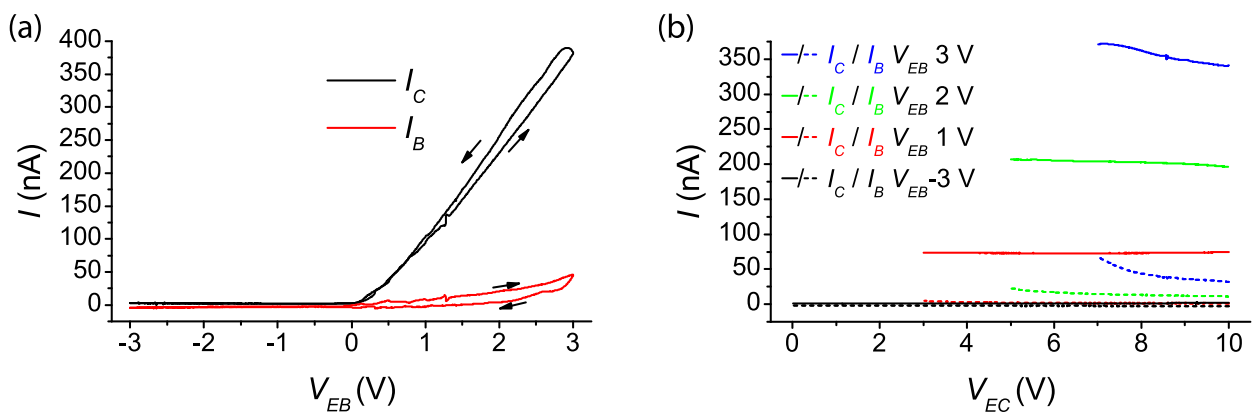

FIG. 3. Transfer and output curves. (a) The transfer curve is low for negative $V_{E B}$ and increases linearly for positive $V_{E B}$ with approximately zero threshold. (b) The output curves show $I_{C}$ saturating with respect of $V_{E C}$ for positive $V_{E B}$. 
current gains are approximately equal to previous IBJTs, ${ }^{12,14,21}$ at above 100 and 10 , respectively. The on-off ratio and ion current gain are more dependent on the selectivity of the membranes and the charge of the junction.

Further, the need to separate the layers in a PEG-IBJT puts high demands on the patterning resolution and alignment accuracy to reduce the separation between emitter/collector and base. As polyphosphonium allows the IBJT to be built without separation of layers, miniaturization of the junction is relatively easier to obtain. The switching speed can potentially be further improved by retaining the base material between the emitter and collector (see Figure 1(b)), thus allowing for a more direct pathway for $I_{\mathrm{C}}$. This design would, however, require a much more accurate layer alignment or that the base patterned on top of the emitter and collector layers. In general, such modifications of device geometry are simpler to accomplish with the non-EFE water dissociating polyphosphonium as fewer active layers are used, suggesting a further use of polyphosphonium to improve switching speed and miniaturization of IBJTs. Such further advancement in IBJT performance would be welcomed, for example, in the continued work towards complex ionic circuits ${ }^{14}$ to regulate signalling in bioelectronics and in drug delivery applications, in which generation of dynamic and complex gradients, at high spatial resolution, is of generic interest.

This research was financed by VINNOVA (OBOE Miljö and AFM), the Swedish Research Council, and the Önnesjö foundation.

${ }^{1}$ R. Karnik, C. H. Duan, K. Castelino, H. Daiguji, and A. Majumdar, Nano Lett. 7, 547 (2007).

${ }^{2}$ O. J. Cayre, T. C. Suk, and O. D. Velev, J. Am. Chem. Soc. 129, 10801 (2007).

${ }^{3}$ W. Guan, R. Fan, and M. A. Reed, Nat. Commun. 2, 506 (2011).

${ }^{4}$ H. Daiguji, Y. Oka, and K. Shirono, Nano Lett. 5, 2274 (2005).

${ }^{5}$ S. W. Nam, M. J. Rooks, K. B. Kim, and S. M. Rossnagel, Nano Lett. 9, 2044 (2009).

${ }^{6}$ R. Karnik, R. Fan, M. Yue, D. Li, P. Yang, and A. Majumdar, Nano Lett. 5, 943 (2005).

${ }^{7}$ C. Zhong, Y. Deng, A. F. Roudsari, A. Kapetanovic, M. P. Anantram, and M. Rolandi, Nat. Commun. 2, 476 (2011).

${ }^{8}$ E. B. Kalman, I. Vlassiouk, and Z. S. Siwy, Adv. Mater. 20, 293 (2008).

${ }^{9}$ J. H. Han, K. B. Kim, H. C. Kim, and T. D. Chung, Angew. Chem., Int. Ed. 48, 3830 (2009).

${ }^{10}$ E. O. Gabrielsson, K. Tybrandt, and M. Berggren, Lab Chip 12, 2507 (2012).

${ }^{11}$ E. O. Gabrielsson and M. Berggren, Biomicrofluidics 7, 064117 (2013).

${ }^{12}$ K. Tybrandt, K. C. Larsson, A. Richter-Dahlfors, and M. Berggren, Proc. Natl. Acad. Sci. U.S.A. 107, 9929 (2010).

${ }^{13}$ K. B. Kim, J. H. Han, H. C. Kim, and T. D. Chung, Appl. Phys. Lett. 96, 143506 (2010).

${ }^{14} \mathrm{~K}$. Tybrandt, R. Forchheimer, and M. Berggren, Nat. Commun. 3, 871 (2012).

${ }^{15}$ V. J. Frilette, J. Phys. Chem. 60, 435 (1956).

${ }^{16}$ S. Mafe and P. Ramirez, Acta Polym. 48, 234 (1997).

${ }^{17}$ A. V. Sokirko, P. Ramirez, J. A. Manzanares, and S. Mafés, Ber. Bunsen Ges. Phys. Chem. 97, 1040 (1993).

${ }^{18}$ B. Lovreček, A. Despić, and J. O. Bockris, J. Phys. Chem. 63, 750 (1959).

${ }^{19}$ S. Mafé, P. Ramirez, and J. A. Manzanares, Berichte Der Bunsen Ges. Phys. Chem. 98, 202 (1994).

${ }^{20}$ H. Strathmann, J. J. Krol, H. J. Rapp, and G. Eigenberger, J. Membr. Sci. 125, 123 (1997).

${ }^{21}$ K. Tybrandt, E. O. Gabrielsson, and M. Berggren, J. Am. Chem. Soc. 133, 10141 (2011).

${ }^{22}$ A. V. Volkov, K. Tybrandt, M. Berggren, and I. V. Zozoulenko, Langmuir 30, 6999 (2014). 